\title{
Carbon ion radiotherapy: impact of tumor differentiation on local control in experimental prostate carcinomas
}

\author{
Christin Glowa ${ }^{1,2,3^{*}}$, Peter Peschke ${ }^{2,3}$, Stephan Brons ${ }^{3,4}$, Oliver C. Neels ${ }^{5,6}$, Klaus Kopka ${ }^{5,6}$, Jürgen Debus $s^{1,3}$ \\ and Christian P. Karger ${ }^{2,3}$
}

\begin{abstract}
Background: To summarize the research activities of the "clinical research group heavy ion therapy", funded by the German Research Foundation (DFG, KFO 214), on the impact of intrinsic tumor characteristics (grading, hypoxia) on local tumor control after carbon $\left({ }^{12} \mathrm{C}-\right)$ ion- and photon irradiations.

Methods: Three sublines of syngeneic rat prostate tumors (R3327) with various differentiation levels (highly $(-H)$, moderately (-HI) or anaplastic (-AT1), (diameter $10 \mathrm{~mm}$ ) were irradiated with 1,2 and 6 fractions of either ${ }^{12} \mathrm{C}$-ions or $6 \mathrm{MV}$ photons using increasing dose levels. Primary endpoint was local tumor control at 300 days. The relative biological effectiveness (RBE) of ${ }^{12} \mathrm{C}$-ions was calculated from $\mathrm{TCD}_{50}$-values (dose at $50 \%$ tumor control probability) of photons and ${ }^{12} \mathrm{C}$-ions and correlated with intrinsic tumor parameters. For the HI-subline, larger tumors (diameter $18 \mathrm{~mm}$ ) were irradiated with either carbon ions, oxygen ions or photons under ambient as well as hypoxic conditions to determine the variability of the RBE under different oxygenation levels. In addition, imaging, histology and molecular analyses were performed to decipher the underlying mechanisms.

Results: Experimental results revealed (i) a smaller variation of the $T C D_{50}$-values between the three tumor sublines for ${ }^{12} \mathrm{C}$-ions (23.6 - $32.9 \mathrm{~Gy}$ ) than for photons (38.2 - 75.7 Gy), (ii) steeper dose-response curves for ${ }^{12} \mathrm{C}$-ions, and (iii) an RBE that increased with tumor grading $(1.62 \pm 0.11(\mathrm{H})$ vs $2.08 \pm 0.13(\mathrm{HI})$ vs $2.30 \pm 0.08$ (AT1)). Large HI-tumors resulted in a marked increase of $\mathrm{TCD}_{50}$, which was increased further by $15 \%$ under hypoxic relative to oxic conditions. Noninvasive imaging, histology and molecular analyses identified hypoxia as an important radioresistance factor in photon therapy.

Conclusions: The dose-response studies revealed a higher efficacy of ${ }^{12} \mathrm{C}$-ions relative to photon therapy in the investigated syngeneic tumor model. Hypoxia turned out to be at least one important radioresistance factor, which can be partly overridden by high-LET ion beams. This might be used to increase treatment effectiveness also in patients.

The results of this project served as a starting point for several ongoing research projects.
\end{abstract}

Keywords: Carbon ion radiotherapy, Relative biological effectiveness (RBE), Prostate tumor, Hypoxia imaging

\section{Background}

Regarding the effectiveness of carbon ion beams, most systematic experimental investigations have been performed in vitro [1-3] or in normal tissue complication models in vivo $[4,5]$ and only very few

\footnotetext{
* Correspondence: c.glowa@dkfz.de

'Department of Radiation Oncology, University Hospital Heidelberg, Im Neuenheimer Feld 400, 69120 Heidelberg, Germany

${ }^{2}$ Department of Medical Physics in Radiation Oncology (E040), German

Cancer Research Center (DKFZ), Im Neuenheimer Feld 280, 69120

Heidelberg, Germany

Full list of author information is available at the end of the article
}

quantitative data on the response of different tumor types to carbon ion beams are currently available [6-9]. As in treatment planning for photon radiotherapy, tumors are still considered as biologically homogeneous entities and the relative biological effectiveness (RBE) is computed by biomathematical models [10-12] to adjust for the dependencies of the RBE on linear energy transfer (LET) and dose. Biological response characteristics of the tumor are described by very few parameters neglecting the impact of additional tumor-associated biological factors 
on the RBE. However, any parameter that influences the tumor response differently for photons and ion beams is expected to influence the RBE. Therefore, to better understand the tumor response to ion beams, these dependencies have to be identified and assessed quantitatively in systematic preclinical experiments.

In previous studies, we determined the dose response curves for the anaplastic prostate carcinoma R2327AT1 $[13,14]$ after 1,2 and 6 fractions of photons and carbon ions, respectively, for the clinically relevant endpoint "local tumor control at 300 days". It was found that the dependence on fractionation was much weaker than for normal tissue [4] resulting in smaller RBE-values at low fractional doses as compared to normal tissue. Although two other tumor types showed similar RBE-values at the same LET $[7,8]$, no systematic investigation of the RBE of tumors varying with respect to growth kinetic and differentiation status have been performed yet.

Within the translationally oriented clinical research group KFO 214 on heavy ion therapy, funded by the German Research Foundation (DFG), the radiation response of a well $(-\mathrm{H})$, moderately $(-\mathrm{HI})$ and poorly (-AT1) differentiated subline of the R2327 prostate carcinoma model was investigated in terms of doseresponse curves for the endpoint "local tumor control at 300 days". As it was found that the radiation responses of the three tumor lines differ much less for carbon ions than for photons, potential influence factors were further investigated by radiological imaging as well as on the histological and molecular level. Furthermore, the relationship between tumor microenvironment and local tumor control and its dependence on radiation quality was evaluated by dedicated irradiation experiments. This contribution gives a brief summary of the overall project and adds to previously published data $[6,15,16]$.

\section{Methods}

\section{Tumor model}

Fresh tumor fragments of the syngeneic Dunning prostate adenocarcinoma sublines $\mathrm{R} 3327-\mathrm{H},-\mathrm{HI}$ and -AT1 [17] were implanted subcutaneously into the distal thigh of male Copenhagen rats (weight 180-200 g, Charles River Laboratories, Wilmington, Massachusetts, USA). During irradiation of $\mathrm{H}$ - and HI-tumors, rats were always kept under inhalation anesthesia with a mixture of $2.5 \%$ sevoflurane (Abbott, Wiesbaden, Germany) and oxygen at $21 / \mathrm{min}$ using an inhalation mask. For AT1irradiations, animals were anesthetized with an intraperitoneal injection of Ketamine hydrochloride $(125 \mathrm{mg} / \mathrm{kg}$, Pfizer Deutschland, Berlin, Germany) mixed with Xylazine hydrochloride $(20 \mathrm{mg} / \mathrm{kg}$, Bayer HealthCare, Leverkusen, Germany) and breathed air [13]. Imaging studies were performed with 3-3.5\% sevoflurane and $1 \mathrm{l} /$ min oxygen. All experiments were approved by the governmental review committee on animal care, and animals were kept under standard laboratory conditions.

\section{Irradiation setup}

The general experimental setup has been described previously $[6,13,14]$. Briefly, for tumor irradiations, rats were placed in a special device for accurate positioning. Tumors of two different sizes were irradiated: Small tumors with a mean diameter at treatment of $10.5 \mathrm{~mm}$ (range 9.0 to $12.0 \mathrm{~mm}$ ) were irradiated with carbon ions at the center of a single $20 \mathrm{~mm}$ SOBP (dose-averaged LET in the tumor: $75 \mathrm{keV} / \mu \mathrm{m}$, range $64-96 \mathrm{keV} / \mu \mathrm{m}$ ) having a field diameter of $18 \mathrm{~mm}$ (90\% isodose). Large tumors had a mean diameter at treatment of $16.5 \mathrm{~mm}$ (range 15.5 to $18.5 \mathrm{~mm}$ ) and were irradiated either with carbon or oxygen ions $\left({ }^{16} \mathrm{O}\right.$-ions $)$ at the center of a single $30 \mathrm{~mm}$ spread-out Bragg-peak (SOBP) (dose-averaged LET in the tumor: $65 \mathrm{keV} / \mu \mathrm{m}$, range $52-91 \mathrm{keV} / \mu \mathrm{m}$ for carbon and $101 \mathrm{keV} / \mu \mathrm{m}$, range $82-142 \mathrm{keV} / \mu \mathrm{m}$ for oxygen ions, respectively) having a field diameter of $25 \mathrm{~mm}$ (90\% isodose). The range of the ions was adjusted by a polymethyl methacrylate (PMMA)-bolus of appropriate thickness. A second PMMA-plate was positioned behind the tumor.

Photon irradiations were performed under identical conditions using a single $6 \mathrm{MV}$ beam of a linear accelerator (Siemens Artiste, Erlangen, Germany) and a PMMA-bolus in front and behind the tumor. Irradiation fields were produced with a cylindrical collimator for the small tumors (90\% isodose: $15 \mathrm{~mm}$ ) and with a multileaf collimator for the larger tumors (90\% isodose: $24 \mathrm{~mm})$, respectively.

\section{Dose response studies}

For small tumors, dose response experiments were performed for all three tumor-sublines (AT1, HI and H) with either 1,2 or 6 fractions using increasing dose levels of either carbon ions or photons. In total, this experimental series contained 859 animals (374 for carbon ions and 405 for photons) including 80 shamtreated controls.

In a second series, large tumors of the HI-subline were treated with single doses under oxic as well as under hypoxic conditions using increasing dose levels of either carbon ions, oxygen ions or photons. Hypoxic conditions were realized by clamping the tumor-supplying artery $10 \mathrm{~min}$ before and during treatment. In total, this experimental series contained 280 animals (45/44 for carbon ions, 37/36 for oxygen ions and 47/48 for photons under oxic/hypoxic conditions); 23 sham-treated animals served as controls.

Following irradiation, tumor volume was measured twice weekly in both experimental series using a caliper. 
Primary endpoint was local tumor control at 300 days, defined as no detectable tumor regrowth. As the $\mathrm{H}$-subline exhibited residual nodules, they were harvested and analyzed histologically for fibrosis (Hematoxylin/Eosin; H\&E) and proliferation 5-bromo-2'-desoxyuridine (BrdU). A fibrotic pattern without proliferation was considered as secondary endpoint for locally controlled H-tumors.

For the primary endpoint, actuarial control rates were calculated and the logistic dose-response model was fitted using the maximum likelihood fitting procedure of the software STATISTICA (version 10.0, Statsoft Inc., www.statsoft.com) (see [6] for details). For the secondary endpoint, no actuarial approach was required as surviving tumor cells were directly detected with a proliferation marker. For both endpoints, the RBE was calculated as the ratio of the $\mathrm{TCD}_{50}$-values (dose at $50 \%$ tumor control probability) for photons and ${ }^{12} \mathrm{C}$-ions.

\section{Positron-Emission-Tomography (PET)}

Dynamic PET measurements with different radiofluorinated 2-nitroimidazole derivatives on a patient scanner (Biograph $^{\mathrm{Tm}}$ mCT, $128 \mathrm{~S}$, Siemens, Erlangen, Germany) were performed to characterize the hypoxic status of small $\left(0.8 \pm 0.5 \mathrm{~cm}^{3}\right)$ and very large $\left(4.4 \pm 2.8 \mathrm{~cm}^{3}\right) \mathrm{H}-, \mathrm{HI}-$ and AT1-tumors prior to irradiation. For this, $15-53 \mathrm{MBq}$ of $\left[{ }^{18} \mathrm{~F}\right]$ fluoromisonidazole $\left(\left[{ }^{18} \mathrm{~F}\right] \mathrm{FMISO}\right)$ were injected into the tail vein of the animals and PET images were recorded over a time period of $60 \mathrm{~min}$ using a 28 -frame protocol (for details, see [16]). In total, this study included 30 tumors (10 AT1, $12 \mathrm{HI}$ and $8 \mathrm{H}$ ).

Additional static measurements in 12 HI-tumors (diameter $16 \mathrm{~mm}$ ) were performed on a PET/CT (Inveon Micro-PET/SPECT/CT, Siemens Medical Solutions, Knoxville, USA) before and 2, 9, and 21d after carbon ion or photon irradiation, respectively. In these measurements, 38-52 MBq $\left[{ }^{18} \mathrm{~F}\right]$ fluoroazomycin arabinoside $\left(\left[{ }^{18} \mathrm{~F}\right] \mathrm{FAZA}\right)$ were administered into the tail vein and images were evaluated at $2 \mathrm{~h}$ post-injection.

\section{T1-weighted dynamic contrast enhanced magnetic resonance imaging (DCE-MRI)}

T1-weighted DCE-MRI measurements were performed in 17 small HI-tumors before as well as 3, 7, 14 and 21 days after single doses (isoeffective doses $18 \mathrm{~Gy}{ }^{12} \mathrm{C}$-ions vs. 37 Gy photons and 37 Gy ${ }^{12} \mathrm{C}$-ions vs. 75 Gy photons, respectively) using a clinical $1.5 \mathrm{~T}$ MRI (Symphony, Siemens, Erlangen, Germany) together with an in-house built small animal coil. Irradiations were carried out either with carbon ions or photons using the same absorbed as well as the same RBE-weighted doses. Each animal had a sham-treated tumor on the contralateral side as internal control.

A T2-weighted turbo spin echo sequence (TR $3240 \mathrm{~ms}$, TE $81 \mathrm{~ms}$, slice thickness $1.5 \mathrm{~mm}$, pixel size
$0.35 \mathrm{~mm}$ ) was used to position the image slice of the DCE-MRI measurement (TR $373 \mathrm{~ms}$, TE 1,67 ms, slice thickness $4.5 \mathrm{~mm}$, pixel size $0.99 \mathrm{~mm}$ ) at the center of the tumor. $30 \mathrm{~s}$ after starting the DCE-MRI measurement, $0.1 \mathrm{mmol} / \mathrm{kg}$ Gd-DTPA (Magnevist', Bayer Healthcare Pharmaceuticals, Berlin, Germany) was injected into the tail vein. Tumor volume and the kinetics of the contrast agent were analyzed using the inhouse software "Medical Imaging Interaction Toolkit" (dkfz, Heidelberg, Germany $[18,19]$ ).

\section{Doppler-Ultrasound imaging}

Ultrasound imaging was performed for 16 small HItumors from different dose-groups of the carbon ion and photon single fraction dose-response studies using a Power Doppler Ultrasound of $30 \mathrm{MHz}$ and the RMV704 transducer (slice thickness $200 \mu \mathrm{m}$, VEVO770, VisualSonics, Toronto, Canada). Animals were measured before and weekly or 2-weekly after irradiation.

\section{Flow cytometric analysis}

DNA-index and cell cycle distribution as well as potential surface stem cell marker of untreated tumors were identified with flow cytometry. Single cell suspensions obtained from frozen tissue were incubated with $2.1 \%$ citric acid including $0.5 \%$ tween 20 and shaking for $20 \mathrm{~min}$ at room temperature. Afterwards, $700 \mu \mathrm{l}$ of the cell suspension supernatant were transferred into a vial, containing $4 \mathrm{ml}$

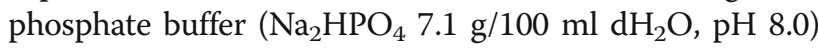
with $2 \mu \mathrm{g} / \mathrm{ml} \mathrm{4}{ }^{\prime}$,6-diamidino-2-phenylindole (DAPI) and analyzed on a PAS II flow cytometer (PARTEC, Münster, Germany). For details see [15]. Cryo-preserved tumor tissue was prepared as single cell suspension using isolation buffer. Afterwards cells were stained for CD24-PE, CD44FITC, CD133-PE, CD326-FITC, cytokeratin 5/8 and 19 labelled with an Alexa Fluor 488 secondary antibody and measured in the Galaxy pro Flow cytometer (PARTEC, Münster, Germany). Flow cytometric analysis was confirmed with staining of cryo-preserved and FFPE tumor tissue (for details, see [15]).

\section{Tumor induction analysis via limiting dilution assay} $\mathrm{CD} 24^{+} / \mathrm{CD} 45^{-}$and $\mathrm{CD} 24^{-} / \mathrm{CD} 45^{-}$untreated AT1-, HIand $\mathrm{H}$-tumor cells were enriched and sorted (FACS Aria, $\mathrm{BD}$, Heidelberg, Germany) from freshly prepared tumor tissue. $500.000 \mathrm{CD} 24^{-} / \mathrm{CD} 45^{-}$cells and various cell numbers between 10 to $200.000 \mathrm{CD} 24^{+} / \mathrm{CD} 45^{-}$cells were injected in a Matrigel suspension (BD, Heidelberg, Germany) subcutaneously into the right and left thigh of animals. The tumor induction was monitored for 300 days.

\section{Histological and molecular studies}

Before and at several time points after single dose irradiation $(8 \mathrm{~h}, 18 \mathrm{~h}, 72 \mathrm{~h}, 7 \mathrm{~d}, 14 \mathrm{~d}, 21 \mathrm{~d})$ tumor tissue was 
cryo-preserved, cut into $7 \mu \mathrm{m}$ cryo-sections (Mikrom HM560, Thermo Fisher Scientific, Dreieich, Germany) and fixed in methanol/acetone for immunofluorescence stainings. Alternatively, formalin-fixed paraffin-embedded (FFPE) tissue was processed with the Microtom (Microm STS Section-Transfer-System, Thermo Fisher Scientific, Dreieich, Germany) and used for H\&E staining.

To analyze the secondary endpoint in the $\mathrm{H}$-tumor, cryo-preserved sections of the residual nodules were stained for proliferating cells using a BrdU antibody (Roche Diagnostics, Mannheim, Germany), which was injected intraperitoneally $(100 \mathrm{mg} / \mathrm{kg}$, Sigma-Aldrich, Taufkirchen, Germany) prior to sacrificing the animal. Vessel architecture (CD31), pericytes (smooth muscle actin) and perfusion as well as hypoxic fraction (pimonidazole) was stained using published protocols $[6,16]$.

For gene expression analysis, HI-tumor tissue was minced in liquid nitrogen using a Potter $\mathrm{S}$ with a Teflon tube extruder (B. Braun, Melsungen, Germany) and RNA was extracted immediately with the NucleoSpin ${ }^{\circ}$ RNA L Kit (Macherey-Nagel, Düren, Germany). RNA-quantity (NanoDrop ${ }^{\circ}$ ND-1000 Peqlab, Erlangen, Germany) and quality (Agilent RNA 6000 Nano Kit and Agilent Bioanalyzer 2100, Agilent, Waldbronn, Germany) were verified. Gene expression profiling was performed according to the manufacturers' protocol (Agilent) using the Whole Rat Genome Kit 4x44k, Low Input Quick Amp Labeling Kit One-Color, gene expression hybridization Kit, RNA-Spike In Kit One-Color, SSPE washing buffer and stabilization and drying solutions.

\section{Results}

Dose-response studies for three different sublines

Figure 1 displays the dose-response curves for the three tumor sublines after single doses of carbon ions or photons, respectively, using the primary endpoint "local control". The $\mathrm{TCD}_{50}$-values were $75.7 \pm 1.6$ (AT1), $62.4 \pm 3.2$ (HI) and $38.2 \pm 1.8(\mathrm{H})$ for photons and $32.9 \pm 0.9$ (AT1), $30.0 \pm 1.1(\mathrm{HI})$ and $23.6 \pm 1.1(\mathrm{H})$ for carbon ions, respectively. The corresponding RBE-values were found to be $2.30 \pm 0.08$ (AT1), $2.08 \pm 0.13(\mathrm{HI})$ and $1.62 \pm 0.11(\mathrm{H})$.

Mean tumor regression (complete tumor volume reduction) times for the AT1-, HI- and $\mathrm{H}$-tumor were $42 \pm 1.7 \mathrm{~d}, 110 \pm 4.7 \mathrm{~d}$ and $\geq 300 \mathrm{~d}$ for photons and $44 \pm 1.7 \mathrm{~d}, 80 \pm 2.0 \mathrm{~d}$ and $\geq 300 \mathrm{~d}$ for ${ }^{12} \mathrm{C}$-ions, respectively. While locally controlled AT1- and HItumors regressed completely, tiny nodules remained in case of locally controlled $\mathrm{H}$-tumors. Lack of proliferative activity associated with a fibrotic tissue pattern used as secondary histological endpoint resulted in an increase of $\mathrm{TCD}_{50}$ of 10.1 Gy for photons but only 3.2 Gy for ${ }^{12} \mathrm{C}$-ions. The corresponding RBE was 1.80 \pm 0.13 .

\section{Structural and functional characterization of the three sublines}

Histological characterization of the three sublines concerning differentiation, hypoxia and vessel density as well as vessel maturity lead to the conclusion that welldifferentiated, slow growing $\mathrm{H}$-tumors have more mature vessels with a minor proportion of hypoxia, whereas HI-tumors are characterized by a more diffuse vascular supply and lack of pericytes in most of their vessels. The AT1-tumor vessels consist of mainly tiny capillary structures causing a volume-dependent higher fraction of hypoxia (Fig. 2).

Dynamic $\left[{ }^{18} \mathrm{~F}\right] \mathrm{FMISO}$ PET of all three untreated tumor sublines showed standardized uptake values $\left(\mathrm{SUV}_{\max }\right)$ of $1.33 \pm 0.52$ in large AT1-tumors and $1.12 \pm 0.83$ in large $\mathrm{HI}$-tumors. In $\mathrm{H}$-tumors no significant tracer uptake was found $\left(\mathrm{SUV}_{\max } 0.63 \pm 0.16\right)$. These characteristics were confirmed by the histological staining with pimonidazole (hypoxic fraction: 62\% (AT1), 54\% (HI) and 7\% (H)). Small tumors did not show a significant uptake at all. The three sublines exhibited differently-shaped time activity curves (TAC). All H-tumors showed a high perfusion-related peak at a few seconds after tracer injection followed by a rapid decrease. HI-tumors reacted much more heterogeneously, with just a small initial peak and a rapid decrease afterwards in most tumors, however, in some HI-tumors a small peak and a positive slope at later time points was found. These two relative similar TAC-shapes could also be detected in the AT1-tumors, however, a third TAC-shape, described by a barely noticeable initial peak and subsequent increasing uptake thereafter was additionally found.

\section{DNA-index, cell cycle analysis and surface marker expression using flow cytometry}

All three tumor sublines gained aneuploidic subpopulations with a high proliferative activity as compared to normal prostate tissue. Ploidy status as well as the number of aneuploidic cells were significantly higher in the AT1-tumors than in the two other tumor lines. In addition, the AT1-tumor always exhibited the largest amount of potential stem cells (i.e. $\mathrm{CD} 24^{+} / \mathrm{CD} 45^{-}$cells). Injecting 100 of these potential stem cells subcutaneously into the hind limb of animals resulted in an induction and complete reproduction of tumors with the same histology for the H- and HI-, but not for the AT1tumor subline. More details on the results of this study can be found in Glowa et al. [15].

\section{Dose-response studies for HI-tumors with and without clamping}

Large HI-tumors were selected for this study because this moderately differentiated tumor exhibited the largest response heterogeneity after photon irradiation. As compared to small tumors, the $\mathrm{TCD}_{50}$-values for the 


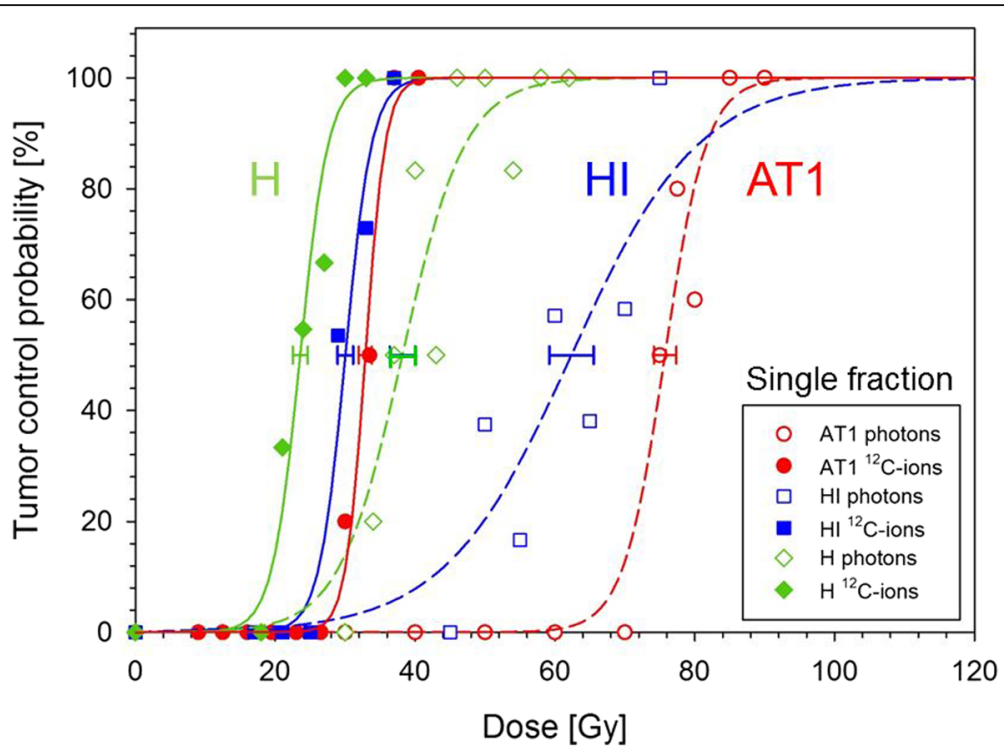

Fig. 1 Dose-response curves of three sublines of the R3327 prostate carcinoma after a single fraction of photons (dashed lines) and ${ }^{12} \mathrm{C}$-ions (solid lines) for the endpoint local tumor control at 300 days, respectively. The uncertainty (1 SD) of TCD 50 is indicated
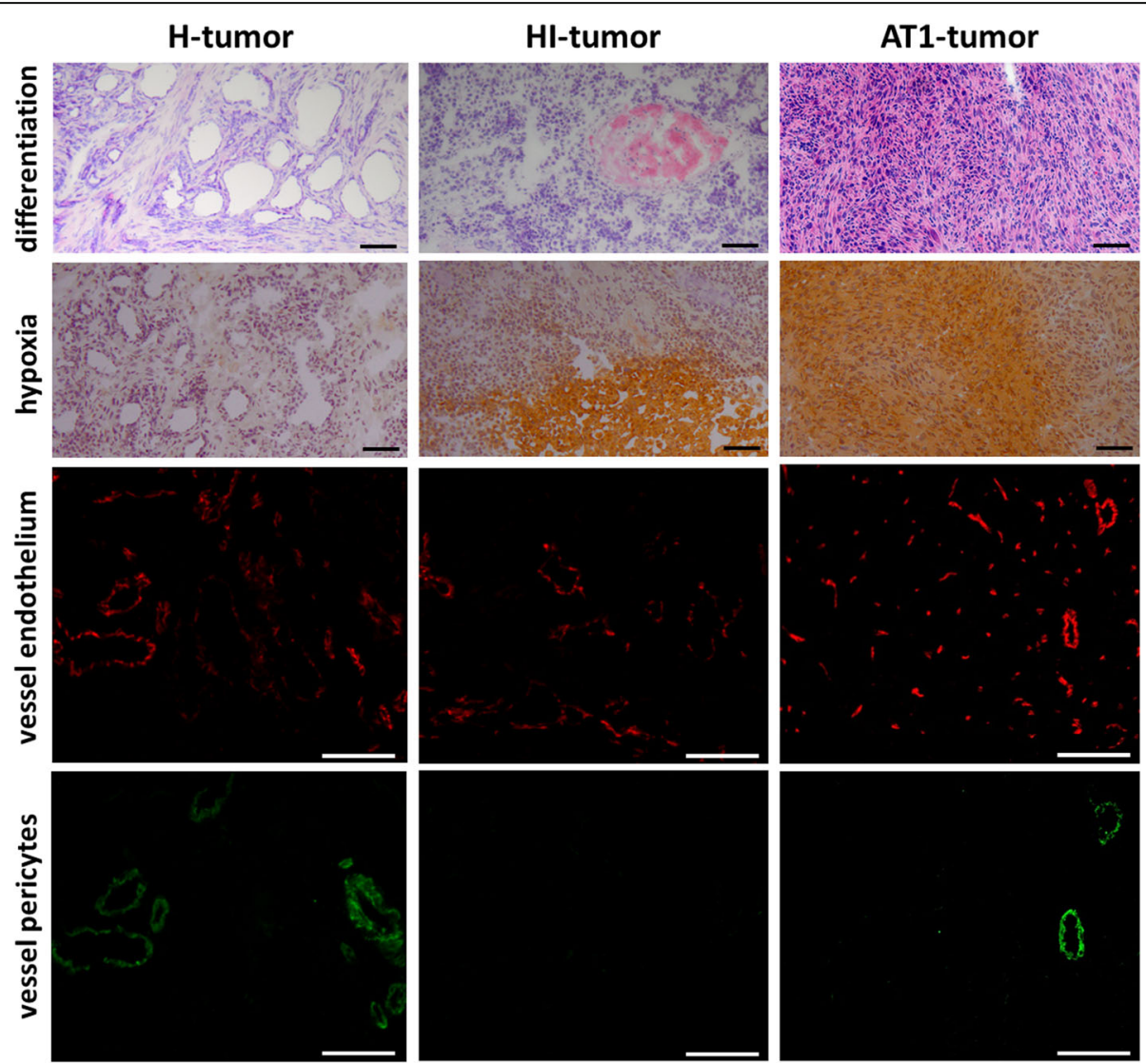

Fig. 2 Comparison of histological sections for the H- (left column), HI- (middle column) and AT1-tumor (right column). Structural changes and differentiation level are detected by Hematoxylin / Eosin (H\&E) staining (upper row). For visualization of hypoxic areas, pimonidazole was used (brown staining, $2^{\text {nd }}$ row) and cell nuclei were counterstained with Hematoxylin. Vessel endothelium was stained with CD31 antibody in red ( $3^{\text {rd }}$ row). The maturity of vessels was proven by a pericyte staining using a smooth muscle actin antibody. Magnification: $100 \times\left(1^{\text {st }}\right.$ and $2^{\text {nd }}$ row) or $200 \times\left(3^{\text {rd }}\right.$ and $4^{\text {th }}$ row). Scale bars are $100 \mu \mathrm{m}$ 
large tumors after single dose irradiations were about $30 \%$ higher under ambient conditions. For the large tumors, the $\mathrm{TCD}_{50}$-value after a single dose of photon irradiation under clamping as compared to ambient conditions was found to be increased by $\sim 15 \%$, while no difference was found for ${ }^{12} \mathrm{C}$-ions and for ${ }^{16} \mathrm{O}$-ions. This, in turn, lead to an increased RBE under hypoxic conditions for both ion types.

Treatment effects after photon and carbon ion irradiation Doppler ultrasound imaging in HI-tumors exhibited a decrease in tumor volume as well as in blood flow after single dose treatment with $33 \mathrm{~Gy}{ }^{12} \mathrm{C}$-ions and 75 Gy photons, respectively. In these locally controlled tumors, the blood flow decreased to an individual tumor baseline and remained constant thereafter (Fig. 3 upper row). The histologically determined high perfusion early after irradiation was well reflected by the ultrasound measurements. However, tumors treated with a single fractions of subtherapeutic doses $\left(21 \mathrm{~Gy}{ }^{12} \mathrm{C}\right.$-ions or 45 Gy photons) showed an increase of blood flow with increasing tumor volume (Fig. 3 lower row). Compared to unirradiated controls, the blood flow decreased after irradiation, but averaged over all animals, there was no significant difference between locally controlled and uncontrolled tumors or between photons and ${ }^{12} \mathrm{C}$-ions in HI-tumors until the onset of clear tumor recurrence.

HI-tumors were irradiated with single doses using either the same physical doses (37 Gy) or isoeffective doses $\left(18 \mathrm{~Gy}{ }^{12} \mathrm{C}\right.$-ions vs. 37 Gy photons and $37 \mathrm{~Gy}{ }^{12} \mathrm{C}$ ions vs. 75 Gy photons) of photons and ${ }^{12} \mathrm{C}$-ions, respectively. Necrosis was identified by combining T2weighted as well as T1-weighted images. After MRI contrast agent injection a more pronounced and faster signal enhancement for both dose levels was seen at 3 and 7 days after irradiation with ${ }^{12} \mathrm{C}$-ions compared to photons (Fig. 4).

Static $\left[{ }^{18} \mathrm{~F}\right]$ FAZA-PET measurements of large HItumors before treatment showed an $\mathrm{SUV}_{\max }$ of up to 1.8 and an increased tumor-to-muscle ratio greater than 3 . Independent of the radiation quality, an apparent complete reoxygenation was found with $\left[{ }^{18} \mathrm{~F}\right]$ FAZA-PET 7 days after single fraction irradiations (Fig. 5).

Histological analysis revealed a shortened latency time for vessel damage, cell cycle arrest and cell death with a concordant prolonged repair time after carbon ions as compared to photons in all three sublines after single fraction irradiation. In addition, inflammatory activity was markedly increased. Gene expression profiling and molecular analysis confirmed these results. Generally, genes involved in DNA-repair, cell cycle arrest, cell-cell interaction and migration as well as cytokines and caspases were overexpressed. Especially the time-dependent induction of genes was extended after carbon ions.

\section{Discussion}

Preclinical studies in normal tissues are preferentially performed to evaluate potential side effects of carbon ions and to validate RBE-models. In contrast, tumor experiments aim to decipher biological factors, which influence the tumor response differently for photons and ion beams, and to identify, which tumor entities might benefit most likely from high-LET irradiations. In this context, a systematic study was initiated to quantitatively assess the treatment response of three different tumor lines to photons and ${ }^{12} \mathrm{C}$-ions using a local tumor control assay.

In summary, the following clinically relevant results (Fig. 1) were obtained: (i) For photons, a considerably heterogeneous treatment response was found, documented by a broad range of $\mathrm{TCD}_{50}$-values for the three tumor-

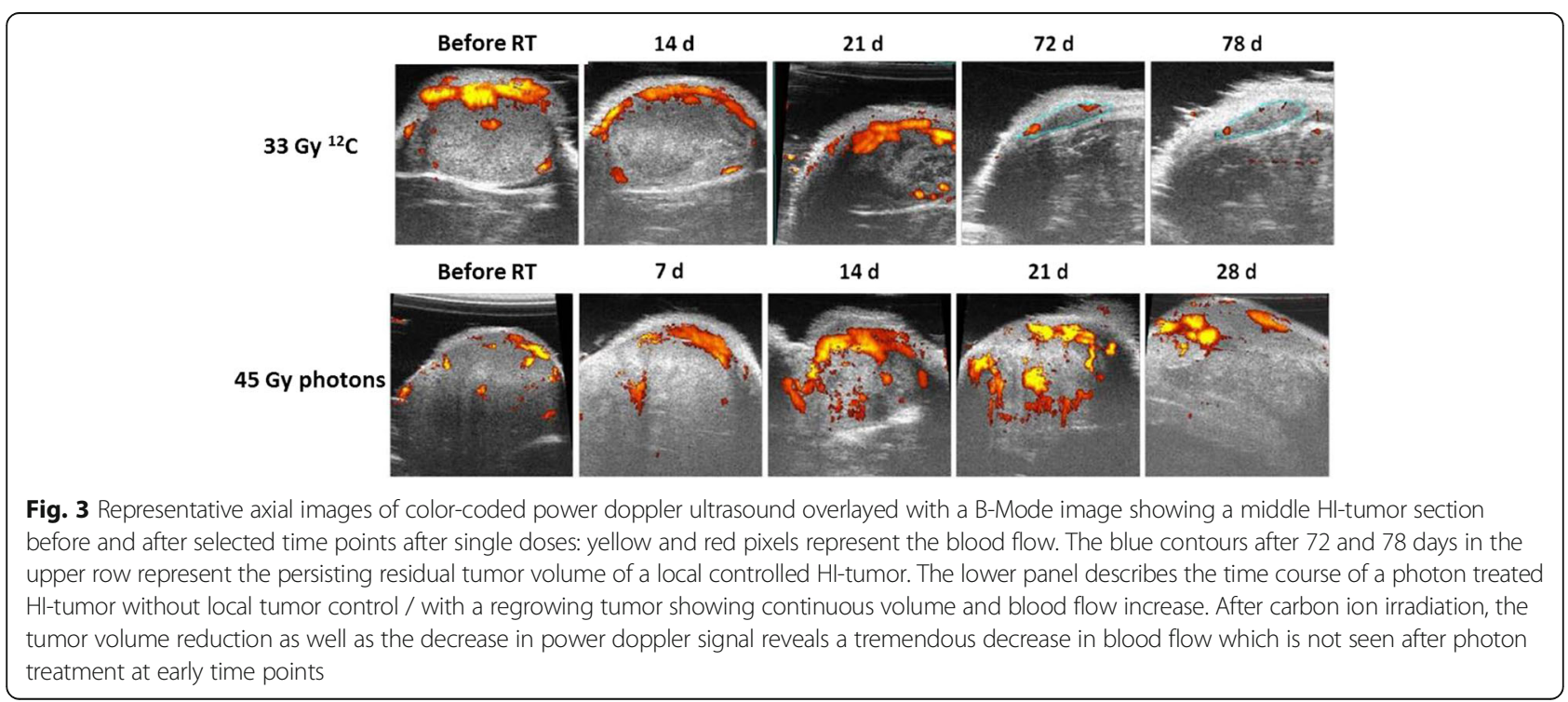




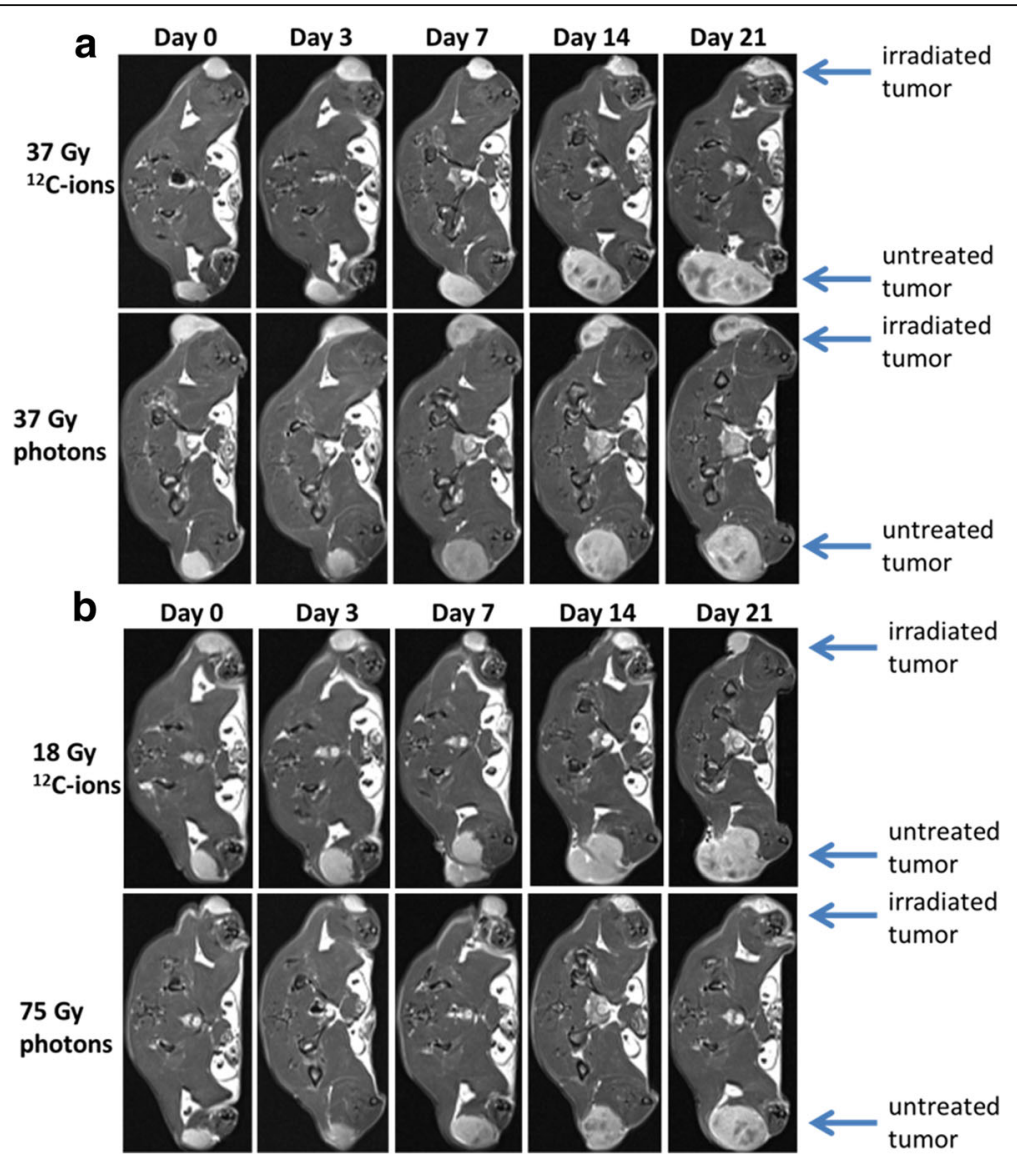

Fig. 4 Exemplarily T1-weighted axial MR-images measured 6 min after contrast agent injection. HI-tumors were either treated (upper tumor in each image) or untreated (lower tumors). Tumors were measured before RT (Day 0) and at 4 time points after single doses. Due to the fast growth of untreated tumors, a longer follow-up was not possible. In (a) $37 \mathrm{~Gy}$ isodoses of photons and ${ }^{12} \mathrm{C}$-ions are compared whereas in (b) isoeffective doses with respect to local control at 300 days are shown ( 18 Gy ${ }^{12} \mathrm{C}$-ions vs. $37 \mathrm{~Gy}$ photons and $37 \mathrm{~Gy}{ }^{12} \mathrm{C}$-ions vs. 75 Gy photons, respectively). A volume increase was seen only in untreated tumors. The light contrast showed edema, whereas dark volumes are a sign for necrotic areas

sublines. (ii) For carbon ions, the respective dose-response curves were located much closer to each other. (iii) In addition, the slope of the dose-response curve for each tumor subline was comparable or steeper for ${ }^{12} \mathrm{C}$-ions than for photons, and (iv) the resulting RBE increased with tumor grading (i.e. H vs. HI vs. AT1). This increase of $\mathrm{RBE}$ predominantly results from a rise of $\mathrm{TCD}_{50}$ with tumor grading in photon treatments while the variation of the treatment response to ${ }^{12} \mathrm{C}$-ions is only small. This supports the conclusion that certain tumor-associated factors might be responsible to render tumors more resistant to photons than to ${ }^{12} \mathrm{C}$-ions. Clearly, these factors are depending on tumor grade. Moreover, also intra-tumoral heterogeneity seems to possess minor impact as documented by the increased slope of the dose-response curve of ${ }^{12} \mathrm{C}$-ions for the very heterogeneous $\mathrm{HI}$-subline as compared to the respective curve for photons. These results allow the conclusion that the response to ${ }^{12} \mathrm{C}$-ions is also less dependent on intra-tumor heterogeneity. Regarding the effectiveness, the highest $\mathrm{RBE}$ of ${ }^{12} \mathrm{C}$-ions can be expected for undifferentiated tumors, showing the highest resistance against photon irradiations. A first report on prostate cancer patients in Japan confirmed our results showing very high tumor control rates with reduced toxicity and a comparable 5-year local control rate for carbon ions between low, intermediate and high-risk prostate cancer patients [20].

From a technical point of view, assessment of local control was most difficult in the slow-growing and well differentiated $\mathrm{H}$-tumor because of frequently occurring residual tissue nodules at the end of the follow-up time. This problem was solved by additional histological analysis using lack of proliferative activity within these nodules as secondary endpoint. Interestingly, as the corresponding $\mathrm{TCD}_{50}$-values increased for both, photons and ${ }^{12} \mathrm{C}$-ions, there was only a minor difference in RBE and the above conclusion remains unchanged [6].

While this report refers to single dose irradiations only, the identical study was conducted for 2 and 6 fractions, already published for the AT1-tumor [14]. Although still 


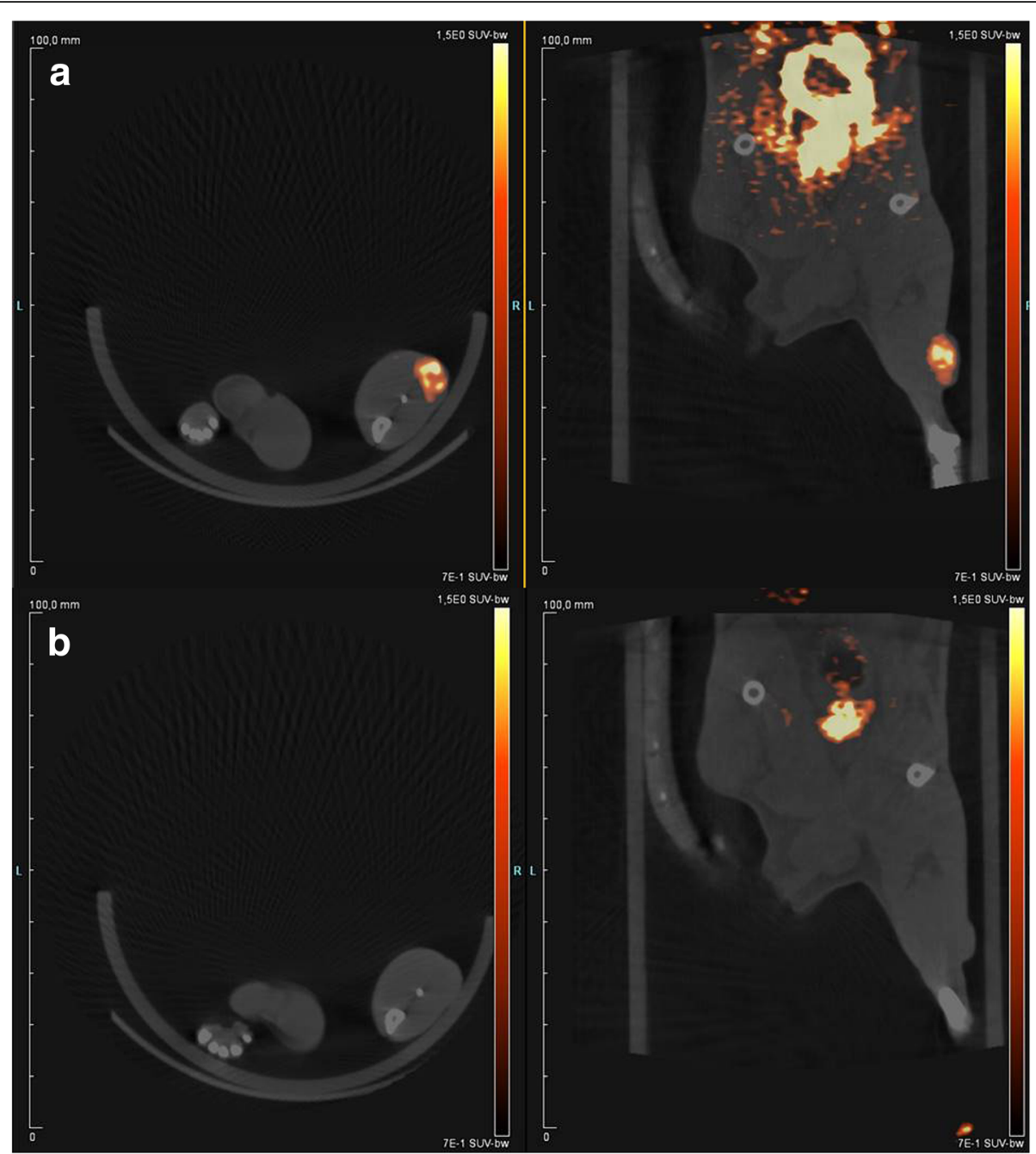

Fig. $5\left[{ }^{18} \mathrm{~F}\right] \mathrm{FAZA}-\mathrm{PET} / \mathrm{CT}$ of a HI-tumor $2 \mathrm{~h}$ post tracer injection, before (a) and after single fraction of ${ }^{12} \mathrm{C}$-ions (b) in axial (left panel) and coronar (right panel) image orientations. The PET tracer is color-coded and overlayed to an aligned CT image. Before RT (a), a high tracer uptake was found in the tumor as well as in the bladder due to partly urinary excretion of the tracer. 7 days after RT (b), the same tumor showed a tracer uptake in the bladder but not in the tumor

under evaluation for the HI- and the H-tumor, there is a clear trend that fractionation increases the $\mathrm{TCD}_{50}$-values in all three tumor cell lines and both irradiation modalities. Again, the shift is larger for photons than for carbon ions, indicating an increasing RBE with decreasing dose per fraction and decreasing differentiation status. The highest RBE for 6 daily fractions $(2.67 \pm 0.15)$ was found for the anaplastic AT1-subline [14]. Details on the complete fractionated studies, including the dose dependence of the RBE and the determination of $\alpha / \beta$-ratios will be published separately. An interesting side observation of the published study [14] was that in the fast growing AT1tumor the metastatic rate increased, when the number of fractions raised from 2 to 6 . Yet, at least for the given treatment schedules (1,2 and 6 fractions) the results were not dependent on radiation quality [21].
There is significant evidence in the literature that resistance to photon therapy is associated with both, intrinsic cellular factors conditioned by the evolutionary capacity of cancer phenotypes as well as epigenetic parameters, or the temporal and spatial heterogeneity of the tumor microenvironment caused by structural abnormalities and density of tumor microvessels, dysfunctional blood flow, low $\mathrm{pH}$ leading to either chronic or acute hypoxic conditions [22-25].

For further clarification, a detailed structural and functional characterization of all three tumor lines prior to irradiation was undertaken. As highly aneuploidic subpopulations were present in all three tumor lines, the ploidic status was not considered as a relevant tumorassociated intrinsic factor for the differential radiation response [15]. In contrast, differences were detected with 
respect to putative cancer stem-like cells characterized as $\mathrm{CD} 24^{+} / \mathrm{CD} 45^{-}$cells, which were positively tested for the ability to form new tumors in a functional limiting dilution assays (Glowa et al., unpublished data). The fact that stem-cell properties were detected in $\mathrm{H}$ - and $\mathrm{HI}$ - but not in AT1-tumors needs further analysis which is presently ongoing.

Dramatic differences were found with respect to the structure and quality of the tumor vascularization and in correlation with the tumor microenvironment, inasmuch as a range of differently oxygenated tumors were detected, with the highest hypoxic fraction in the poorly differentiated AT1-tumors and nearly no detectable hypoxia in the well differentiated $\mathrm{H}$-tumors. These results are in line with a previous report on the same tumor model using TOLDMRI [10]. In addition, the $\left[{ }^{18} \mathrm{~F}\right] \mathrm{FMISO}-\mathrm{TAC}$-curves in PET were extremely variable between the three tumor sublines indicating also large differences in perfusion [5]. Thus, the investigated tumor-sublines represent a wide range of differently oxygenated tumors allowing dedicated investigation of the role of oxygenation on the radiation response.

To further exploit the role of ${ }^{12} \mathrm{C}$-ions to overcome hypoxia, which is presumably the most important resistance factor in photon therapy, a four-armed dose-response study was performed. Larger moderately differentiated HI-tumors were selected as model tumors because of its proven hypoxia and its extensive heterogeneous treatment response to photons. For larger HI-tumors treated with photons either under ambient or complete hypoxic (clamping) conditions, the detected oxygen enhancement ratio (OER) was clearly below 2 , which is in line with previously published in vivo studies [26, 27]. Generally, OERs for single dose irradiations in solid tumors under clamping conditions were found to be lower than in cell culture studies [3,28], presumably because tumor cells in intact tissues are not only impacted by the intrinsic cellular radioresistance but also by additional factors like cell to cell communication, bystander effects, and the immune response. Moreover, clamping does not only create a transient severe hypoxic state but also reduces the nutrient supply and induces a strong extracellular pressure to the capillaries which might increase secondary tumor cell death and therefore masks the potentially higher OER to some degree. When ${ }^{12} \mathrm{C}$-ions (dose average LET: $65 \mathrm{keV} /$ $\mu \mathrm{m})$ were applied under identical experimental conditions an up to $15 \%$ lower OER was found for larger HI-tumors. The detected decrease of OER for ${ }^{12} \mathrm{C}$-ions is relevant and if confirmed in patients would increase effectiveness dramatically. With this respect, the only available study, which compares the impact of tumor oxygenation for ${ }^{12} \mathrm{C}$ ions and photons in patients, is inconclusive [29].

A detailed comparison with the previous doseresponse experiments for the small tumors, however, turned out to be difficult as the $\mathrm{TCD}_{50}$-values after photon and ${ }^{12} \mathrm{C}$-ion irradiations under non-clamping conditions were found to be substantially higher as compared to the previously investigated small tumors. This suggests that larger tumors are not only associated with an increased number of tumor cells but also that volumedependent alterations of the tumor micromilieu might play a role. To investigate this hypothesis in more detail, the clamping experiments are currently repeated for the small tumors within a new project and a comparison of the response of small and large tumors will be published separately when the results are available.

Radioresistance of tumors due to hypoxia is clinically of highest relevance as oxic tumors have a much higher disease-free survival than hypoxic tumors, as has been shown in head and neck cancer patients [30]. Therefore, the observed reduction of the OER for ${ }^{12} \mathrm{C}$-ions is a very important finding for the treatment of hypoxic tumors. In a first patient cohort treated with carbon ions, Japanese colleagues verified a smaller OER of ${ }^{12} \mathrm{C}$-ions in uterine cancer [29] and our findings in the experimental prostate carcinomas confirm this, however, further analyses are necessary. Although the obtained promising OERs for ${ }^{12} \mathrm{C}$-ions might in principal be used to overcome radioresistance evoked by severe hypoxia, the dependence of OER on LET is still an open question. In vitro the OER for high LET irradiations decreases with increasing LET and is expected to be negligible at LETs higher than $200 \mathrm{keV} / \mu \mathrm{m}$ [31]. Our preliminary results based on doseresponse studies with oxygen ions (dose average LET: $101 \mathrm{keV} / \mu \mathrm{m}$ ) using the same tumor model also indicates a small OER near to 1.

Finally, structural and functional assessments were performed in HI-tumors to decipher some of the biological mechanisms, responsible for the differential effects of ${ }^{12} \mathrm{C}$ ions and photons. In-house synthetized $\left[{ }^{18} \mathrm{~F}\right] \mathrm{FAZA}$ in combination with static PET measurements in a dedicated small animal scanner (Inveon Micro-PET/SPECT/CT (Siemens Medical Solutions, Knoxville, USA)) has been established in larger HI-tumors. A significant traceruptake prior to treatment followed by a clear reduction 1 week after irradiation was found for photons as well as for ${ }^{12} \mathrm{C}$-ions in a first pilot study. The hypoxia imaging tracer $\left[{ }^{18} \mathrm{~F}\right] \mathrm{FAZA}$ is a well-established alternative to the first generation tracer $\left[{ }^{18} \mathrm{~F}\right] \mathrm{FMISO}$ and is known to correlate well with both, autoradiography and the hypoxia marker pimonidazole [32]. Yet, results so far are reported to be ambivalent. No significant general reoxygenation was seen for $\mathrm{SiHa}$ cervix tumors in mice after fractionated irradiations with 10 or 25 Gy photons [32], while reoxygenation has been shown after 2 weeks of fractionated radiotherapy for patients with head and neck cancer in some but not in all cases [33]. Radiation-induced reoxygenation seems to depend on tumor type and on the intrinsic characteristics of 
the individual tumor. Tumor cell inactivation, tissue shrinkage, vessel damage and altered perfusion are biological factors associated with oxygenation changes after irradiation. A very striking observation was the extremely fast emerging of vascular disruptions already $18 \mathrm{~h}$ after single doses of carbon ions, while similar changes were delayed after photon irradiations. Nevertheless, a clear correlation between vessel integrity and hypoxia or reoxygenation is currently missing.

In spite of existing vascular structures, oxygen delivery to neoplastic and stromal cells is frequently reduced or even abolished by increased vessel distances, severe structural abnormalities of tumor microvessels, disturbed microcirculation and increased interstitial pressure. This can lead to false negative results in PET measurements as the tracer may not reach the hypoxic regions. To independently assess functional microenvironmental disturbations, measurements with doppler ultrasound as well as DCE-MRI were performed. Ultrasound measurements revealed a high blood flow followed by a rapid and dramatic decrease after carbon ions and later a steady state in the first 4 weeks after irradiation. Besides, this initial effect no other significant variation was detected between the two radiation modalities as well as for different dose levels. A further refinement of these results is expected from photoacoustic imaging [34], which allows assessing the oxygen saturation of tumor vessels based on optical detection of oxy- and deoxyhemoglobin. Similar to the ultrasound measurements, preliminary results of DCE-MRI revealed a faster and higher contrast enhancement after single doses of ${ }^{12} \mathrm{C}$-ions as compared to photons, which is in line with the more prominent vessel damage observed on the histological level. This first assessment is based on a qualitative rather than quantitative data analysis and a more detailed investigation using pharmacokinetic modeling to extract perfusion-related tissue parameters is ongoing.

\section{Conclusions}

Although the picture of the differential response to photon and ${ }^{12} \mathrm{C}$-ion irradiation is not yet fully completed, the described radiobiological research program has systematically documented the higher efficacy of ${ }^{12} \mathrm{C}$-ion therapy in a syngeneic experimental tumor model. It has been shown that tumor-associated resistance factors can be partly overridden by high-LET ion beam therapy. Moreover, the results provide evidence that the increased effectiveness of high-LET radiation is related to tumor-associated factors, preferentially those which are responsible to render tumors more resistant to conventional photon treatments. Dose-response studies revealed that hypoxia is one of the dominant radioresistance factors and that its impact can be reduced by high-LET ion beams on a clinically relevant level. This could potentially improve the probability of local tumor control relative to conventional photon treatments. Pronounced vascular disruptions associated with enhanced cell inactivation together with a rapid disturbance of the tumor microenvironment were identified as the most striking mechanistic effects of carbon ions and these results served as starting point for various ongoing research projects.

\section{Abbreviations \\ $\left[{ }^{18} \mathrm{~F}\right] \mathrm{FAZA}:\left[{ }^{18} \mathrm{~F}\right]$ fluoroazomycin arabinoside; $\left[{ }^{18} \mathrm{~F}\right] \mathrm{FMISO}:\left[{ }^{18} \mathrm{~F}\right]$ fluoromisonidazole; ${ }^{12} \mathrm{C}$-ions: Carbon ions; ${ }^{16} \mathrm{O}$-ions: Oxygen ions; BrdU: Bromodesoxyuridine; DCE-MRI: Dynamic contrast enhanced magnetic resonance imaging; DFG: German Research Foundation; FFPE: Formalin-fixed paraffin-embedded; H\&E: Hematoxylin/Eosin staining; LET: Linear energy transfer; OER: Oxygen enhancement ratio; PET: Positron-Emission-Tomography; PMMA: Polymethyl methacrylate; RBE: Relative biological effectiveness; SOBP: Spread-out Bragg-peak; SUV: Standard uptake value; TAC: Time activity curve; $\mathrm{TCD}_{50}$-value: Irradiation dose with $50 \%$ tumor control probability}

\section{Acknowledgements}

The authors are grateful to Prof. Eric Hahn for his valuable hints in experiment design and the staff of the Heidelberg lon Therapy Center (HIT) for providing excellent working conditions. The support of the DKFZ Core Facilities, the Centers for preclinical research and small animal imaging, are greatly acknowledged. Especially the technical support of Inna Babushkina and Victoria Eichwald for in vivo imaging and Yvonne Remde for $\left[{ }^{18} \mathrm{~F}\right] \mathrm{FAZA}$ synthesis is very much appreciated. We thank PD Volker Ehemann for cell cycle analysis and surface marker expression detection via FACS. This work was supported by the German Research Foundation (DFG, KFO 214).

\section{Funding}

This study was supported by the German Research Foundation (DFG, KFO 214).

\section{Availability of data and materials}

The data generated and analyzed during the current study are available from the corresponding author on reasonable request.

\section{Authors' contributions}

CG carried out all experiments and analyzes and was a major contributor in writing of the manuscript; PP initiated the project, was involved in study design and data analyses as well as writing the manuscript; SB supported the high-LET irradiations at HIT; OCN and KK established the $\left[{ }^{18} \mathrm{~F}\right] \mathrm{FAZA}$-PET synthetization and produced the tracer for the experiments; JD initiated the project and was involved in the translational assessment of the acquired data; CPK initiated the project and was involved in study design, data analyses, dosimetry, treatment planning, statistical evaluation and writing of the manuscript. All authors read and approved the final manuscript.

\section{Ethics approval}

All experiments were approved by the governmental review committee on animal care (G171/09, G43/11, G116/11, G182/11, G88/14), and animals were kept under standard laboratory conditions.

\section{Consent for publication}

Not applicable

\section{Competing interests}

The authors declare that they have no competing interests.

\section{Publisher's Note}

Springer Nature remains neutral with regard to jurisdictional claims in published maps and institutional affiliations.

\section{Author details}

'Department of Radiation Oncology, University Hospital Heidelberg, Im Neuenheimer Feld 400, 69120 Heidelberg, Germany. ${ }^{2}$ Department of Medical Physics in Radiation Oncology (E040), German Cancer Research Center 
(DKFZ), Im Neuenheimer Feld 280, 69120 Heidelberg, Germany. ${ }^{3}$ National Center for Radiation Research in Oncology (NCRO), Heidelberg Institute for Radiation Oncology (HIRO), Im Neuenheimer Feld 400, 69120 Heidelberg, Germany. ${ }^{4}$ Heidelberg Ion Beam Therapy Center (HIT), Im Neuenheimer Feld 280, 69120 Heidelberg, Germany. ${ }^{5}$ Division of Radiopharmaceutical Chemistry, German Cancer Research Center (DKFZ), Im Neuenheimer Feld 280, 69120 Heidelberg, Germany. ${ }^{6}$ German Cancer Consortium (DKTK), Im Neuenheimer Feld 400, 69120 Heidelberg, Germany.

Received: 3 August 2017 Accepted: 27 October 2017

Published online: 09 November 2017

\section{References}

1. Weyrather WK, Ritter S, Scholz M, Kraft G. RBE for carbon track-segment irradiation in cell lines of differing repair capacity. Int J Radiat Biol. 1999;75: 1357-64.

2. Suzuki M, Kase $Y$, Yamaguchi H, Kanai T, Ando K. Relative biological effectiveness for cell-killing effect on various human cell lines irradiated with heavy-ion medical accelerator in Chiba (HIMAC) carbon-ion beams. Int J Radiat Oncol Biol Phys. 2000;48:241-50.

3. Furusawa Y, Fukutsu K, Aoki M, Itsukaichi H, Eguchi-Kasai K, Ohara H, Yatagai $F$, Kanai T, Ando K. Inactivation of aerobic and hypoxic cells from three different cell lines by accelerated (3)He-, (12) C- and (20)Ne-ion beams. Radiat Res. 2000;154:485-96.

4. Karger CP, Peschke P, Sanchez-Brandelik R, Scholz M, Debus J. Radiation tolerance of the rat spinal cord after 6 and 18 fractions of photons and carbon ions: experimental results and clinical implications. Int J Radiat Oncol Biol Phys. 2006;66:1488-97.

5. Saager M, Glowa C, Peschke P, Brons S, Grun R, Scholz M, Huber PE, Debus J, Karger CP. Split dose carbon ion irradiation of the rat spinal cord: dependence of the relative biological effectiveness on dose and linear energy transfer. Radiother Oncol. 2015;117:358-63.

6. Glowa C, Karger CP, Brons S, Zhao D, Mason RP, Huber PE, Debus J, Peschke P. Carbon ion radiotherapy decreases the impact of tumor heterogeneity on radiation response in experimental prostate tumors. Cancer Lett. 2016;378:97-103.

7. Koike S, Ando K, Oohira C, Fukawa T, Lee R, Takai N, Monobe M, Furusawa Y, Aoki M, Yamada S, Shimizu W, Nojima K, Majima H. Relative biological effectiveness of $290 \mathrm{MeV} / \mathrm{u}$ carbon ions for the growth delay of a radioresistant murine fibrosarcoma. J Radiat Res. 2002:43:247-55.

8. Tenforde TS, Tenforde SD, Crabtree KE, Parks DL, Schilling WA, Parr SS, Flynn MJ, Howard J, Lyman JT, Curtis SB. RBE values for radiation-induced growth delay in rat rhabdomyosarcoma tumors exposed to plateau and peak carbon, neon and argon ions. Int J Radiat Oncol Biol Phys. 1981;7:217-22.

9. Sorensen BS, Horsman MR, Alsner J, Overgaard J, Durante M, Scholz M, Friedrich T, Bassler N. Relative biological effectiveness of carbon ions for tumor control, acute skin damage and late radiation-induced fibrosis in a mouse model. Acta Oncol. 2015;54:1623-30

10. Scholz M, Kellerer AM, Kraft-Weyrather W, Kraft G. Computation of cell survival in heavy ion beams for therapy. The model and its approximation. Radiat Environ Biophys. 1997;36:59-66

11. Kanai T, Furusawa Y, Fukutsu K, Itsukaichi H, Eguchi-Kasai K, Ohara H. Irradiation of mixed beam and design of spread-out Bragg peak for heavyion radiotherapy. Radiat Res. 1997;147:78-85.

12. Inaniwa T, Kanematsu N. A trichrome beam model for biological dose calculation in scanned carbon-ion radiotherapy treatment planning. Phys Med Biol. 2015;60:437-51.

13. Peschke P, Karger CP, Scholz M, Debus J, Huber PE. Relative biological effectiveness of carbon ions for local tumor control of a radioresistant prostate carcinoma in the rat. Int J Radiat Oncol Biol Phys. 2011;79:239-46.

14. Karger CP, Peschke P, Scholz M, Huber PE, Debus J. Relative biological effectiveness of carbon ions in a rat prostate carcinoma in vivo: comparison of 1, 2, and 6 fractions. Int J Radiat Oncol Biol Phys. 2013;86:450-5.

15. Glowa C, Peschke P, Karger CP, Hahn EW, Huber PE, Debus J, Ehemann V. Flow cytometric characterization of tumor subpopulations in three sublines of the dunning R3327 rat prostate tumor model. Prostate. 2013;73:1710-20.

16. Mena-Romano P, Cheng C, Glowa C, Peschke P, Pan L, Haberkorn U, Dimitrakopoulou-Strauss A, Karger CP. Measurement of hypoxia-related parameters in three sublines of a rat prostate carcinoma using dynamic (18)F-FMISO-PET-CT and quantitative histology. Am J Nucl Med Mol Imaging. 2015;5:348-62.
17. Isaacs JT, Heston WD, Weissman RM, Coffey DS. Animal models of the hormone-sensitive and -insensitive prostatic adenocarcinomas, dunning R3327-H, R-3327-HI, and R-3327-AT. Cancer Res. 1978;38:4353-9.

18. Nolden M, Zelzer S, Seitel A, Wald D, Muller M, Franz AM, Maleike D, Fangerau M, Baumhauer M, Maier-Hein L, Maier-Hein KH, Meinzer HP, Wolf I. The medical imaging interaction toolkit: challenges and advances : 10 years of open-source development. Int J Comput Assist Radiol Surg. 2013;8:607-20.

19. Debus $C$, Floca $R$, Norenberg $D$, Abdollahi A, Ingrisch M. Impact of fitting algorithms on errors of parameter estimates in dynamic contrast enhanced MRI. Phys Med Biol. 2017; doi: 10.1088/1361-6560/aa8989.

20. Nomiya T, Tsuji H, Kawamura H, Ohno T, Toyama S, Shioyama Y, Nakayama Y, Nemoto K, Tsujii H, Kamada T. A multi-institutional analysis of prospective studies of carbon ion radiotherapy for prostate cancer: a report from the Japan Carbon ion Radiation Oncology Study Group (J-CROS). Radiother Oncol. 2016:121:288-93.

21. Karger CP, Scholz M, Huber PE, Debus J, Peschke P. Photon and carbon ion irradiation of a rat prostate carcinoma: does a higher fraction number increase the metastatic rate? Radiat Res. 2014;181:623-8.

22. Tatum JL, Kelloff GJ, Gillies RJ, Arbeit JM, Brown JM, Chao KS, et al. Hypoxia: importance in tumor biology, noninvasive measurement by imaging, and value of its measurement in the management of cancer therapy. Int J Radiat Biol. 2006:82:699-757.

23. Junttila MR, de Sauvage FJ. Influence of tumour micro-environment heterogeneity on therapeutic response. Nature. 2013;501:346-54.

24. Good JS, Harrington KJ. The hallmarks of cancer and the radiation oncologist: updating the 5Rs of radiobiology. Clin Oncol. 2013;25:569-77.

25. Nomiya T, Nemoto K, Miyachi H, Fujimoto K, Takeda K, Ogawa Y, Takai Y, Yamada S. Relationships between radiosensitivity and microvascular density in esophageal carcinoma: significance of hypoxic fraction. Int J Radiat Oncol Biol Phys. 2004:58:589-96.

26. Yaromina A, Thames H, Zhou X, Hering S, Eicheler W, Dorfler A, Leichtner T, Zips D, Baumann M. Radiobiological hypoxia, histological parameters of tumour microenvironment and local tumour control after fractionated irradiation. Radiother Oncol. 2010;96:116-22.

27. Hirayama R, Uzawa A, Takase N, Matsumoto Y, Noguchi M, Koda K, Ozaki M, Yamashita K, Li H, Kase Y, Matsufuji N, Koike S, Masunaga S, Ando K, Okayasu R, Furusawa Y. Evaluation of SCCVII tumor cell survival in clamped and non-clamped solid tumors exposed to carbon-ion beams in comparison to X-rays. Mutat Res. 2013;756:146-51.

28. Wenzl T, Wilkens JJ. Theoretical analysis of the dose dependence of the oxygen enhancement ratio and its relevance for clinical applications. Radiat Oncol. 2011;6:171.

29. Nakano T, Suzuki Y, Ohno T, Kato S, Suzuki M, Morita S, Sato S, Oka K, Tsujii $\mathrm{H}$. Carbon beam therapy overcomes the radiation resistance of uterine cervical cancer originating from hypoxia. Clin Cancer Res. 2006;12:2185-90.

30. Mortensen LS, Johansen J, Kallehauge J, Primdahl H, Busk M, Lassen P, et al. FAZA PET/CT hypoxia imaging in patients with squamous cell carcinoma of the head and neck treated with radiotherapy: results from the DAHANCA 24 trial. Radiother Oncol. 2012:105:14-20.

31. Antonovic L, Brahme A, Furusawa Y, Toma-Dasu I. Radiobiological description of the LET dependence of the cell survival of oxic and anoxic cells irradiated by carbon ions. J Radiat Res. 2013;54:18-26.

32. Busk M, Mortensen LS, Nordsmark M, Overgaard J, Jakobsen S, Hansen KV, Theil J, Kallehauge JF, D'Andrea FP, Steiniche T, Horsman MR. PET hypoxia imaging with FAZA: reproducibility at baseline and during fractionated radiotherapy in tumour-bearing mice. Eur J Nucl Med Mol Imaging. 2013;40:186-97.

33. Zips D, Zophel K, Abolmaali N, Perrin R, Abramyuk A, Haase R, et al. Exploratory prospective trial of hypoxia-specific PET imaging during radiochemotherapy in patients with locally advanced head-and-neck cancer. Radiother Oncol, 2012:105:21-8.

34. Needles A, Heinmiller A, Sun J, Theodoropoulos C, Bates D, Hirson D, Yin M, Foster FS. Development and initial application of a fully integrated photoacoustic micro-ultrasound system. IEEE Trans Ultrason Ferroelectr Freq Control. 2013;60:888-97. 